TRANSACTIONS OF THE

AMERICAN MATHEMATICAL SOCIETY

Volume 348, Number 4, April 1996

\title{
EPIGRAPHICAL AND UNIFORM CONVERGENCE OF CONVEX FUNCTIONS
}

\author{
JONATHAN M. BORWEIN AND JON D. VANDERWERFF
}

\begin{abstract}
We examine when a sequence of lsc convex functions on a Banach space converges uniformly on bounded sets (resp. compact sets) provided it converges Attouch-Wets (resp. Painlevé-Kuratowski). We also obtain related results for pointwise convergence and uniform convergence on weakly compact sets. Some known results concerning the convergence of sequences of linear functionals are shown to also hold for lsc convex functions. For example, a sequence of lsc convex functions converges uniformly on bounded sets to a continuous affine function provided that the convergence is uniform on weakly compact sets and the space does not contain an isomorphic copy of $\ell_{1}$.
\end{abstract}

\section{INTRODUCTION}

In recent years, there have been several papers showing the utility of certain forms of "epi-convergence" in the study of optimization of convex functions. In this area, Attouch-Wets convergence has been shown to be particularly important (we say the sequence of lsc functions $\left\{f_{n}\right\}$ converges Attouch-Wets to $f$ if $d\left(\cdot\right.$, epi $\left.f_{n}\right)$ converges uniformly to $d(\cdot$, epi $f)$ on bounded subsets of $X \times \mathbb{R}$, where for example $d(\cdot$, epi $f)$ denotes the distance function to the epigraph of $f$ ). The name Attouch-Wets is associated with this form of convergence because of the important contributions in its study made by Attouch and Wets. See [1] for one of their several contributions and Beer's book ([3]) and its references for a more complete list. Several applications of Attouch-Wets convergence in convex optimization can be found in Beer and Lucchetti ([8]); see also [3]. Some very recent results concerning Attouch-Wets convergence in the study of subgradient mappings of nonconvex functions have been obtained by Levy et al. in [17].

In this note, one of our principal interests is the connection between AttouchWets convergence and uniform convergence on bounded sets, and with analogous relationships regarding the weaker forms of epi-convergence introduced in the next section. We will only be concerned with lsc convex functions defined on real Banach spaces. There are many results in this direction. For example, one consequence of Salinetti and Wets ([21]) is that Attouch-Wets convergence of lsc convex functions neither implies nor is implied by pointwise convergence. In [7], Beer and DiConcilio establish connections between Attouch-Wets convergence of graphs of

Received by the editors January 17, 1995 and, in revised form, April 3, 1995.

1991 Mathematics Subject Classification. Primary 46A55, 46B20, 52A41.

Key words and phrases. Epi-convergence, lsc convex function, uniform convergence, pointwise convergence, Attouch-Wets convergence, Painlevé-Kuratowski convergence, Mosco convergence.

The first author's research supported in part by an NSERC research grant and by the Shrum endowment 
general lsc functions and uniform convergence on bounded sets. In particular, they observe that for nonconvex functions on metric spaces, Attouch-Wets convergence of graphs does not imply uniform convergence on bounded sets even if the limit function is uniformly continuous on bounded sets (which as they note is contrary to the intuition one might obtain from the Hausdorff versus uniform convergence case). Surprisingly, there does not appear to be any work addressing whether such examples can occur in the convex case. However, in later work, Beer showed that if $\left\{f_{n}\right\}$ is a sequence of lsc convex functions eventually uniformly bounded on bounded sets and if $\left\{f_{n}\right\}$ converges Attouch-Wets to $f$, then $\left\{f_{n}\right\}$ converges to $f$ uniformly on bounded sets ([5, Lemma 4.1]). This result was needed in his characterization of Attouch-Wets convergence via Lipschitz regularizations ([5, Theorem 4.3]).

The preceding result of Beer concerning uniform convergence on bounded sets does not address the situation where only the limit function is assumed to be bounded on bounded sets. In the second section, we will show that Attouch-Wets convergence does indeed imply uniform convergence on bounded sets when the functions are lsc convex and the limit function is bounded on bounded sets - thus in the convex case, the previously mentioned example of [7] cannot arise. Note, it is easy to see that $f$ is bounded on bounded sets provided $\left\{f_{n}\right\}$ is eventually uniformly bounded on bounded sets; see Lemma 1.3(a). We will also prove analogous results for some weaker forms of convergence, including Painlevé-Kuratowski, versus uniform convergence on weakly compact sets, compact sets, singletons, etc. In contrast to our result concerning Attouch-Wets convergence, we show that some eventual uniform boundedness assumption on the sequence of functions is needed even to obtain pointwise convergence from these weaker forms of epi-convergence. Let us mention, for example, that Lahrache used results of this flavor for pointwise convergence in order to derive a result concerning the stability of slice convergence under addition (see [16]) which was used by Beer to provide a characterization of slice convergence in terms of Lipschitz regularizations ([5]).

The last result of the second section, shows that in spaces not containing $\ell_{1}$, uniform convergence on weakly compact sets can be deduced from a form of epiconvergence intermediate to Attouch-Wets and Painlevé-Kuratowski convergence with only a boundedness assumption on the original function (whereas a boundedness condition is needed, for example, when the functions are defined on $\ell_{1}$ ). This is because Mackey and norm convergence coincide sequentially in the dual space of any space not containing $\ell_{1}$ (see $[10,19]$ ). This leads us to the third section where we study when a sequence of convex functions converging uniformly on weakly compact sets (resp. pointwise) converges uniformly on bounded sets (resp. weakly compact sets) to a continuous affine limit function. Indeed, we show that the nonparenthetical result holds in spaces not containing $\ell_{1}$ whereas the parenthetical result holds precisely when Mackey and weak-star convergence agree sequentially in the dual space. Thus we provide a convex function extension of the theorem in [19], and we recapture (without Šmulyan's criterion) some differentiability results of [10]. Unfortunately, as examples show, these results do not extend in a fashion to allow one to obtain Attouch-Wets convergence from a natural weaker form of epi-convergence in spaces not containing $\ell_{1}$.

Our notation is rather standard. The continuous dual of a space $X$ is denoted by $X^{*}$. The closed unit ball of $X$ will be denoted by $B_{X}$. By the Mackey topology on $X^{*}$, we mean the topology of uniform convergence on weakly compact subsets of 
$X$. When we speak of lower semicontinuous (lsc) convex functions, we always mean extended real-valued lsc convex functions that are somewhere finite. A useful property of lsc convex functions, that we will sometimes use, is that they are bounded below on bounded sets. This follows for instance, because for a given lsc convex function $f$, the separation theorem ensures there is a translate of some continuous (hence Lipschitz) linear functional that lies below $f$ (see [20, Proposition 3.15]).

\section{Definitions and Preliminary Lemmas on Epi-Convergence}

For a Banach space, we will let $\mathcal{S}$ denote any one of the following collection of sets: $\mathcal{P}$ the single points, $\mathcal{K}$ the norm compact sets, $\mathcal{W}$ the weakly compact sets, $\mathcal{B}$ the bounded sets.

Definition. We will say a net of closed convex sets $\left\{A_{\nu}\right\} \mathcal{S}$-converges to a closed convex set $A$ if for each $\mathcal{S}$-set $W$ there is a $\bar{\nu}$ such that

$$
W \cap A_{\nu} \subset A+\epsilon B_{X} \text { and } W \cap A \subset A_{\nu}+\epsilon B_{X} \text { for all } \nu \geq \bar{\nu}
$$

If $f_{\nu}$ and $f$ are lsc convex functions on $X$, we will say $\left\{f_{\nu}\right\} \mathcal{S}$-converges to $f$, if $\left\{\right.$ epi $\left.f_{\nu}\right\} \mathcal{S}$-converges to epif in $X \times \mathbb{R}$ endowed with the $\infty$-product of the norms.

Equivalently $\left\{f_{\nu}\right\} \mathcal{S}$-converges to $f$, if for any $\mathcal{S}$-set $W$ in $X$ and any $\mathcal{S}$-interval $I \subset \mathbb{R}$, and $\epsilon>0$, there is a $\bar{\nu}$ such that whenever $\nu \geq \bar{\nu}$ and $w \in W$ one has:

(i) if $f_{\nu}(w) \in I$, then there exists $x \in X$ such that $\|x-w\| \leq \epsilon$ and $\mid f_{\nu}(w)-$ $f(x) \mid \leq \epsilon$;

(ii) if $f(w) \in I$, then there exist $x \in X$ such that $\|x-w\| \leq \epsilon$ and $\left|f_{\nu}(x)-f(w)\right| \leq$ $\epsilon$.

Let us also recall that $\left\{A_{n}\right\}$ is said to converge Painlevé-Kuratowski (resp. Mosco) to $A$, if for each $a \in A$ there exist $a_{n} \in A_{n}$ with $\left\{a_{n}\right\} \rightarrow a$ and if $a \in A$ whenever $\left\{a_{n_{k}}\right\}_{k}$ converges in norm (resp. weakly) to $a$ where $a_{n_{k}} \in A_{n_{k}}$; see [3] for more information.

Observe that $\mathcal{S}$-convergence is invariant under equivalent renorming, and thus our choice to work with the $\infty$-product of the norms on the product space is merely preference of convenience. We don't know if $\mathcal{P}$-convergence or $\mathcal{W}$-convergence have been studied much, if at all (see [22] for classifications and descriptions of many of the known forms of set convergence). Our first remark indicates that $\mathcal{K}$-convergence and $\mathcal{B}$-convergence are well-known forms of set convergence.

Remark 1.1. (a) $\mathcal{B}$-convergence is precisely Attouch-Wets convergence.

(b) For sequences, $\mathcal{K}$-convergence is precisely Painlevé-Kuratowski convergence.

(c) $\mathcal{W}$-convergence implies Mosco convergence but not conversely.

Proof. Part (a) was shown in [2, Lemma 3.1]. We leave (b) as a straightforward exercise since it is not crucial to our development. We also omit the details of (c), although in Remark 3.6 we will show Mosco convergence is strictly weaker $\mathcal{W}$-convergence in any non-Schur space.

Because of this remark, we will often use the terms Painlevé-Kuratowski or Attouch-Wets when speaking of $\mathcal{K}$-convergence or $\mathcal{B}$-convergence. However, we have chosen not to attach specific names to the other forms of set convergence we discuss, because one can view $\mathcal{W}$-convergence, for example, as a variation of Painlevé-Kuratowski, Attouch-Wets or Hausdorff convergence (not to mention the 
several authors that discovered or standardized the formulation of $\mathcal{B}$-convergence as an alternative description of Attouch-Wets convergence - see [5, p. 42]). The next example shows that in general $\mathcal{P}$-convergence may be very weak. However, we will later obtain results showing in many cases it is as strong as Painlevé-Kuratowski convergence.

Remark 1.2. In $\mathbb{R}^{2}$ consider $A=\{(0,0)\}$ and $A_{n}=\left\{(u, v): v=\frac{1}{n} u,|u| \geq \frac{1}{n}\right\}$. Then $\left\{A_{n}\right\} \mathcal{P}$-converges but does not $\mathcal{K}$-converge to $A$. Let $f=I_{A}$ and $f_{n}=I_{A_{n}}-1$ (where $I_{S}$ denotes the indicator function of $S$ ). Then $\liminf _{n \rightarrow \infty} f_{n}\left(\frac{1}{n}, \frac{1}{n^{2}}\right)=-1<$ $f(0,0)$ and $\left\{f_{n}\right\} \mathcal{P}$-converges to $f$ but does not $\mathcal{K}$-converge to $f$. In fact it is wellknown that $f(x) \leq \liminf _{n \rightarrow \infty} f_{n}\left(x_{n}\right)$ whenever $\left\{f_{n}\right\} \mathcal{K}$-converges $f$ and $\left\{x_{n}\right\} \rightarrow x$.

We leave the verification of the previous remark to the reader. However, we will include the proof of the following simple facts which we will use later.

Lemma 1.3. Suppose $f_{\nu}, f$ are lsc convex functions on a Banach space.

(a) If $\left\{f_{\nu}\right\} \mathcal{P}$-converges to $f$, then $f(x) \leq \liminf _{\nu} f_{\nu}(x)$ for all $x \in X$.

(b) If $\left\{f_{\nu}\right\} \mathcal{S}$-converges to $f$, then $\left\{f_{\nu}\right\}$ is eventually uniformly bounded below on each $\mathcal{S}$-set.

(c) If $\left\{f_{n}\right\} \mathcal{P}$-converges to $f$ and $\left\{f_{n}\right\}$ is not eventually bounded below on $\mathcal{S}$-sets, then $\left\{f_{n}\right\}$ is not eventually bounded above on $\mathcal{S}$-sets.

Proof. (a) Suppose $f(w)>\alpha>\liminf _{\nu} f_{\nu}(w)$ for some $w$. Let $r>0$ be such that $\alpha+r<f(w)$. By the lower semicontinuity of $f$, we find $\delta>0$ such that $f(x)>\alpha+\frac{r}{2}$ for $\|x-w\|<\delta$. Let $0<\epsilon<\min \left\{\delta, \frac{r}{2}\right\}$. Then $(w, \alpha) \in \operatorname{epi} f_{\nu^{\prime}}$ for some subnet, while $(w, \alpha) \notin$ epi $f+\epsilon B_{X \times \mathbb{R}}$. This shows $\left\{f_{\nu}\right\}$ does not $\mathcal{P}$-converge to $f$.

(b) Because $f$ is lsc and convex, it is bounded below on bounded sets. Suppose for $\left\{w_{\nu}\right\} \subset W$ an $\mathcal{S}$-set we have $\liminf f_{\nu}\left(w_{\nu}\right)=-\infty$. Let $r<$ $\inf \left\{f(x): x \in W+B_{X}\right\}$. Now $\left(w_{\nu^{\prime}}, r-2\right) \in \operatorname{epi} f_{\nu^{\prime}}^{\nu} \cap(W \times\{r-2\})$ for some subnet, while $\left(w_{\nu}, r-2\right) \notin$ epi $f+B_{X \times \mathbb{R}}$ for all $\nu$. This contradicts the $\mathcal{S}$-convergence of $\left\{f_{\nu}\right\}$ to $f$.

(c) By (b) we know $\mathcal{S}$ cannot be the bornology of singletons. By passing to a subsequence we find a set $W \in \mathcal{S}$ such that $f_{k}\left(w_{k}\right) \rightarrow-\infty$ where $w_{k} \in W$. Fix a sequence $\left\{x_{k}\right\} \rightarrow 0$. Then there is an $r \in \mathbb{R}$ with $f\left(x_{k}\right)>r$ for all $k$. Now the subsequence $\left\{f_{k}\right\} \mathcal{P}$-converges to $f$. So by part (a), we can choose $j_{k} \rightarrow \infty$ such that $f_{j_{k}}\left(x_{k}\right)>r$. By convexity, $\frac{1}{2} f_{j_{k}}\left(2 x_{k}-w_{j_{k}}\right)+\frac{1}{2} f_{j_{k}}\left(w_{j_{k}}\right) \geq r$ for all $k$ and so $\limsup f_{j_{k}}\left(2 x_{k}-w_{j_{k}}\right) \rightarrow \infty$. Because $\left\{2 x_{k}\right\}$ is relatively compact, $\left\{2 x_{k}-w_{j_{k}}\right\}_{k}$ is $k \rightarrow \infty$ an $\mathcal{S}$-set on which $\left\{f_{n}\right\}$ is not eventually uniformly bounded above.

One goal of this note is to determine the relationship between $\mathcal{S}$-convergence and uniform convergence on $\mathcal{S}$-sets. The next implication is known, for example, in the Attouch-Wets case (see [3]).

Lemma 1.4. Suppose $f_{\nu}, f$ are lsc convex functions on a Banach space such that dom $_{\nu} \subset$ domf eventually. If $\left\{f_{\nu}\right\}$ converges uniformly to $f$ on $W \cap$ domf for each $W \in \mathcal{S}$, then $\left\{f_{\nu}\right\} \mathcal{S}$-converges to $f$.

Proof. Fix an arbitrary $\epsilon>0$, and let $A:=\operatorname{epif}$ and $A_{\nu}:=\operatorname{epi} f_{\nu}$. Let $\widetilde{W}$ be any $\mathcal{S}$-set of $X \times \mathbb{R}$. Let $W$ be the projection of $\widetilde{W}$ onto $X$; then $W$ is an $\mathcal{S}$-set. Choose 
$\bar{\nu}$ such that for $\nu \geq \bar{\nu}, \operatorname{dom} f_{\nu} \subset \operatorname{dom} f$ and

$$
\left|f_{\nu}(x)-f(x)\right|<\epsilon \text { for all } x \in W \cap \operatorname{dom} f .
$$

Now suppose $(w, t) \in \widetilde{W} \cap A_{\nu}$ for some $\nu \geq \bar{\nu}$. Then $t \geq f_{\nu}(w)>f(w)-\epsilon$ and so $(w, t) \in A+\epsilon B_{X \times \mathbb{R}}$. Similarly, if $(w, t) \in \widetilde{W} \cap A$, then $t \geq f(w)>f_{\nu}(w)-\epsilon$ for all $\nu \geq \bar{\nu}$ and so $(w, t) \in A_{\nu}+\epsilon B_{X \times \mathbb{R}}$ for all $\nu \geq \bar{\nu}$.

Trivially, if $f=I_{B_{X}}$ and $f_{n}=I_{\left(1-\frac{1}{n}\right) B_{X}}$, then $\left\{f_{n}\right\}$ converges Attouch-Wets but not pointwise to $f$. However the converse of Lemma 1.4 holds in many cases as we will discuss. Variants of the following result are known for Attouch-Wets and some other forms of convergence (see $[3,16])$.

Lemma 1.5. Suppose $\left\{f_{\nu}\right\}$ and $f$ are lsc convex functions on a Banach space $X$. Suppose $W$ is a $\mathcal{S}$-set such that for some $\delta>0$ there are a $\bar{\nu}$ and an $M>0$ such that $f_{\nu}(x) \leq M$ for all $x \in W+\delta B_{X}$ and $\nu \geq \bar{\nu}$. If $\left\{f_{\nu}\right\} \mathcal{S}$-converges to $f$, then $\left\{f_{\nu}\right\}$ converges uniformly to $f$ on $W+\eta B_{X}$ for $\eta<\delta$.

Proof. By Lemma 1.3(a) we know $f(x) \leq M$ for all $x \in W+\delta B_{X}$. Because $\mathcal{S}$ sets are bounded and because $f$ is lsc convex, we know there is an $M_{1} \geq M$ such that $f(x) \geq-M_{1}$ for all $x \in W+\delta B_{X}$. Now suppose $\left\{f_{\nu}\right\}$ does not converge uniformly to $f$ on $W+\eta B_{X}$. Then there is an $\epsilon>0$ such that for any $\tilde{\nu}$ we can find $\nu_{1} \geq \tilde{\nu}$ and $x \in W+\eta B_{X}$ with $f_{\nu_{1}}(x)-f(x)>\epsilon\left(\right.$ or $\left.f_{\nu_{1}}(x)-f(x)<-\epsilon\right)$. In the first case, using this with $\mathcal{S}$-convergence, we choose $\nu_{1} \geq \bar{\nu}, w \in W+\eta B_{X}$ and $\|h\|<\epsilon(\delta-\eta) /\left(6 M_{1}\right)$ such that $f_{\nu_{1}}(w)-f(w)>\epsilon$ and $f_{\nu_{1}}(w+h) \leq f(w)+\frac{\epsilon}{2}$. Let $t=\frac{6 M_{1}}{\epsilon}$. Then $\|t h\|<\delta-\eta$ and by convexity

$$
\frac{1}{t+1} f_{\nu_{1}}(w-t h)+\frac{t}{t+1} f_{\nu_{1}}(w+h) \geq f_{\nu_{1}}(w) ; \text { and thus }
$$

$f_{\nu_{1}}(w-t h) \geq f_{\nu_{1}}(w)+t\left[f_{\nu_{1}}(w)-f_{\nu_{1}}(w+h)\right] \geq f_{\nu_{1}}(w)+t \frac{\epsilon}{2} \geq-M_{1}+3 M_{1}>M$ (since $\left.f_{\nu_{1}}(w) \geq f(w) \geq-M_{1}\right)$. Because $\nu_{1} \geq \bar{\nu}$, this contradicts the boundedness hypothesis on $W+\delta B_{X}$ (in the case $f(w)-f_{\nu_{1}}(w)>\epsilon$ one argues similarly to contradict the boundedness of $f$ on $\left.W+\delta B_{X}\right)$.

The following useful fact was proved by Beer in [5, Lemma 4.1] for the AttouchWets case (i.e. for $\mathcal{S}:=\mathcal{B}$ ); see also [3, Proposition 7.1.3].

Lemma 1.6. Suppose $f_{n}, f$ are lsc convex functions on a Banach space and $\left\{f_{n}\right\}$ is eventually uniformly bounded above on $\widetilde{\mathcal{S}}$-sets where $\widetilde{\mathcal{S}}:=\mathcal{S} \cup \mathcal{K}$. If $\left\{f_{n}\right\} \mathcal{S}$ converges to $f$, then $\left\{f_{n}\right\}$ converges uniformly to $f$ on $\widetilde{\mathcal{S}}$-sets.

Proof. First let us show that for any $\mathcal{S}$-set $W$, there is a $\delta>0$ such that $\left\{f_{n}\right\}$ is eventually uniformly bounded above on $W+\delta B_{X}$. Suppose this is not true for some $\mathcal{S}$-set $W$. For $\delta=\frac{1}{n}$ we find $k \geq n$ and $x_{k}, y_{k} \in W+\frac{1}{n} B_{X}$ with $f_{k}\left(y_{k}\right)-f_{k}\left(x_{k}\right)>$ $k^{2}\left\|x_{k}-y_{k}\right\|$. Now let $h_{k}=y_{k}-x_{k}$ and $t_{k}=\frac{1}{k\left\|h_{k}\right\|}$. By convexity (as in the previous lemma), $f_{k}\left(y_{k}+t_{k} h_{k}\right) \geq f_{k}\left(y_{k}\right)+t_{k}\left[f_{k}\left(y_{k}\right)-f_{k}\left(y_{k}-h_{k}\right)\right]=f_{k}\left(y_{k}\right)+$ $t_{k}\left[f\left(y_{k}\right)-f\left(x_{k}\right)\right] \geq f_{k}\left(y_{k}\right)+k$. Therefore $\left\{f_{k}\right\}$ is not eventually uniformly bounded above on $S:=\left\{y_{k}\right\}_{k} \cup\left\{y_{k}+t_{k} h_{k}\right\}_{k}$. Observe that $S \subset W+K$ for some compact set $K$ because $\left\{t_{k} h_{k}\right\} \rightarrow 0$ and for some $\left\{v_{k}\right\} \rightarrow 0, y_{k}+v_{k} \in W$. Thus $S$ is an $\widetilde{\mathcal{S}}$-set. According to Lemma $1.3(\mathrm{c})$ we know that $\left\{f_{n}\right\}$ is not eventually uniformly bounded 
above on some $\widetilde{\mathcal{S}}$-set. Now for any $\mathcal{S}$ containing the compact sets, $\widetilde{\mathcal{S}}=\mathcal{S}$ and so the conclusion of the lemma follows from Lemma 1.5. If $\mathcal{S}:=\mathcal{P}$, then by Lemma 1.5 we can find a neighborhood around each point on which the convergence is uniform and so a standard argument shows the convergence is uniform on compact sets.

\section{2. $\mathcal{S}$-Convergence And Uniform CONVERGENCE on $\mathcal{S}$-SETS}

Having the necessary preliminaries from the previous section, we are ready for the central result of this section.

Theorem 2.1. Suppose $X$ is a Banach space, $f$ and $f_{n}$ are lsc convex functions and let $B$ be a translate of the unit ball of an equivalent norm on $X$. Then:

(a) If $\left\{f_{n}\right\}$ converges Attouch-Wets to $f$ and $f$ is bounded above on $B+\delta B_{X}$ for some $\delta>0$, then $\left\{f_{n}\right\}$ converges uniformly to $f$ on $B$.

(b) If $\limsup _{n \rightarrow \infty} f_{n}(x)<\infty$ for all $x \in X, f$ is bounded above on $\mathcal{S}$-sets and $\left\{f_{n}\right\}$ $\mathcal{S}$-converges to $f$ where $\mathcal{S}$ contains the compact sets, then $\left\{f_{n}\right\}$ converges uniformly to $f$ on $\mathcal{S}$-sets.

(c) If for some $\delta>0$, $\limsup _{n \rightarrow \infty} f_{n}(x)<\infty$ for all $x \in B+\delta B_{X}$, and if $\left\{f_{n}\right\}$ $\mathcal{P}$-converges to $f$, then $\left\{f_{n}\right\}$ converges uniformly to $f$ on compact subsets of $B$.

Proof. (a) First observe that by translating and renorming we may assume that $B=B_{X}$, the unit ball on $X$, and by replacing $\delta$ with an appropriate smaller number, we have $f$ bounded on $(1+4 \delta) B_{X}$. We will proceed by contradiction. Let us assume that $\left\{f_{n}\right\}$ is not eventually bounded above on $S:=(1+\delta) B_{X}$. Then by passing to a subsequence we choose $x_{k} \in S$ such that $f_{k}\left(x_{k}\right)>k$ for each $k$. Now we let $C_{k}=\left\{x: f_{k}(x) \leq k\right\}$. Then each $C_{k}$ is a closed convex set and $x_{k} \notin C_{k}$. By the separation theorem we choose $\Lambda_{k} \in S_{X^{*}}$ such that

$$
\Lambda_{k}\left(x_{k}\right) \geq \sup _{C_{k}} \Lambda_{k}
$$

Because $\left\{\Lambda_{k}\right\}$ is a sequence of norm one functionals, we can choose $w_{k} \in W:=$ $(1+3 \delta) B_{X}$ such that $\Lambda_{k}\left(w_{k}\right)>1+2 \delta=\sup \{\|x\|: x \in S\}+\delta \geq \sup _{k}\left\{\Lambda_{k}\left(x_{k}\right)\right\}+\delta$. It follows from $(2.1)$ that $d\left(w_{k}, C_{k}\right) \geq \delta$. Therefore,

$$
f_{k}(x)>k \text { whenever }\left\|x-w_{k}\right\|<\delta .
$$

By the hypothesis there is an $\alpha$ such that $f(x) \leq \alpha$ for all $x \in W$. Consider $\widetilde{W}=W \times\{\alpha\} \subset X \times \mathbb{R}$. Clearly $\left(w_{k}, \alpha\right) \in$ epif $\cap \widetilde{W}$. If $\left\|x-w_{k}\right\|<\delta$ and $k \geq \alpha+\delta$, then $(2.2)$ shows $f_{k}(x)>k \geq \alpha+\delta$. Whence $\left(w_{k}, \alpha\right) \notin$ epi $f_{k}+r B_{X \times \mathbb{R}}$ for any $0<r<\delta$ and $k \geq \alpha+\delta$. This contradicts the Attouch-Wets convergence of $\left\{f_{n}\right\}$ to $f$. Therefore the sequence $\left\{f_{n}\right\}$ is eventually uniformly bounded above on $(1+\delta) B_{X}$ and so by Lemma 1.5 the convergence is uniform on $B$.

(b) This proof begins the same way as (a). We outline the details. Suppose $\left\{f_{n}\right\}$ is not eventually uniformly bounded above on some $\mathcal{S}$-set $S$. We choose $x_{k} \in S, C_{k}$ and $\Lambda_{k}$ as before. If $\left\{\Lambda_{k}\right\}$ does not converge uniformly to 0 on $\mathcal{S}$-sets, one chooses an $\mathcal{S}$-set $W$ and passes to a further subsequence if necessary so that $\sup \Lambda_{k}>r+\delta$ where $r=\sup \{\|x\|: x \in S\}$ and $\delta>0$ is fixed; then choose $w_{k} \in W$ so that $\Lambda_{k}\left(w_{k}\right)>r+\delta$. The argument above then shows $\left\{f_{n}\right\}$ does not $\mathcal{S}$-converge to 
$f$. Consequently, $\left\{f_{n}\right\}$ is eventually uniformly bounded above on $\mathcal{S}$-sets provided $\left\{\Lambda_{k}\right\}$ does not converge uniformly to 0 on $\mathcal{S}$-sets.

We now verify $\left\{\Lambda_{k}\right\}$ does not converge uniformly to 0 on $\mathcal{S}$-sets. Indeed, using the condition $\limsup _{n \rightarrow \infty} f_{n}(x)<\infty$ for all $x$ with the Baire category theorem, we find $v \in X$ and positive numbers $\delta, K, N$ such that $f_{n}(x) \leq K$ for $n \geq N$ and $x \in v+\delta B_{X}$. Now for some $K^{\prime} \geq K, N^{\prime} \geq N$ we have $f_{n}(-v) \leq K^{\prime}$ for all $n \geq N^{\prime}$. By convexity $f_{n}(x) \leq K^{\prime}$ for all $n \geq N^{\prime}$ and $\|x\| \leq \frac{\delta}{2}$. This with (2.1) shows $\Lambda_{k}\left(x_{k}\right) \geq \frac{\delta}{2}$ for all $k \geq N^{\prime}$. Because $S$ is a $\mathcal{S}$-set we know $\left\{\Lambda_{k}\right\}$ does not converge to 0 uniformly on $\mathcal{S}$-sets. Thus the previous paragraph shows $\left\{f_{n}\right\}$ is eventually uniformly bounded above on $\mathcal{S}$-sets, so we may apply Lemma 1.6 to complete the proof of (b).

(c) A standard convexity with Baire category argument as used in the previous paragraph shows that $\left\{f_{n}\right\}$ is locally bounded at each point in $B+\frac{\delta}{2} B_{X}$. Hence a standard compactness argument shows that Lemma 1.5 applies. (c)

The next two corollaries record some global consequences of Theorem 2.2(a) and

Corollary 2.2. A proper lsc convex function $f$ on a Banach space is bounded on bounded sets if and only if each sequence of lsc convex functions converging Attouch-Wets to $f$ converges uniformly on bounded sets to $f$.

Proof. The "only if" implication follows from Theorem 2.1(a). For the converse implication, we proceed by contraposition. Let $g_{n}$ be the indicator function of $\frac{1}{n} B_{X}$ and let $f_{n}$ be the function whose epigraph is the closure of the sum of the epigraphs of $f$ and $g_{n}$ (that is, $f_{n}$ is the closure of the infimal convolution of $f$ and $g_{n}$ ). It is geometrically clear that $f_{n}$ converges Attouch-Wets to $f$ (more rigorously, one can apply [3, Theorem 7.3.8]). Let $W$ be a bounded set intersecting the domain of $f$ on which $f$ is unbounded. Because $f$ is unbounded on $W$, we can choose $v_{n}, w_{n} \in W$ with $\left\|v_{n}-w_{n}\right\|<\frac{1}{n}$ but $f\left(w_{n}\right)-f\left(v_{n}\right) \rightarrow \infty$. Write $w_{n}=v_{n}+h_{n}$ where $\left\|h_{n}\right\|<\frac{1}{n}$ and so $f_{n}\left(w_{n}\right) \leq f_{n}\left(v_{n}\right)+g_{n}\left(h_{n}\right)=f\left(v_{n}\right)$ which shows $f\left(w_{n}\right)-f_{n}\left(w_{n}\right) \rightarrow \infty$. Hence we have $\left\{f_{n}\right\}$ converging Attouch-Wets to $f$, but not uniformly on bounded sets.

For comparison, recall that Beer's [5, Theorem 4.3] shows for sufficiently large parameters, the Lipschitz regularizations of $f_{n}$ converge uniformly on bounded sets to the Lipschitz regularization of $f$ if and only if the original sequence converges Attouch-Wets. Let us also mention that [3, Theorem 7.3.8] shows that a "regularizing family of smoothing kernels" of a lsc convex function (see [3, p. 252]) always converges Attouch-Wets and pointwise to the function. Thus from Theorem 2.1 we know that on many sets the convergence is uniform (not just pointwise).

Corollary 2.3. Suppose $f_{n}, f$ are lsc convex functions with $\limsup _{n \rightarrow \infty} f_{n}(x)<\infty$ for all $x$ in a Banach space $X$. Then the following are equivalent.

(a) $\left\{f_{n}\right\}$ converges uniformly to $f$ on compact sets.

(b) $\left\{f_{n}\right\}$ converges Painlevé-Kuratowski to $f$.

(c) $\left\{f_{n}\right\} \mathcal{P}$-converges to $f$.

Proof. According to Lemma 1.3(a) we know $f$ is real valued for all $x$. Applying Lemma 1.4 shows (a) $\Rightarrow(\mathrm{b})$. It is clear that $(\mathrm{b}) \Rightarrow$ (c) follows from the definitions 
and the fact $\mathcal{K}$-convergence is Painlevé-Kuratowski convergence. The nontrivial implication (c) $\Rightarrow$ (a) follows from Theorem 2.1.

Because the topology on the closed convex sets generated by Attouch-Wets convergence is metrizable, there is no need to talk about nets in Theorem 2.1(a). In the next proposition we will show that Theorem 2.1(b) and (c) can fail for nets, and moreover, one cannot remove the condition $\limsup f_{n}(x)<\infty$ for all $x \in X$. In particular this will show the above corollary will fail completely without the limsup condition, because Remark 1.2 has already shown (c) will not necessarily imply (b) even when $X=\mathbb{R}^{2}$. Recall that a Schur space is one in which weak sequential and norm convergence agree (e.g. $\left.\ell_{1}\right)$.

Proposition 2.4. (a) Let $X$ be a (Schur) Banach space with separable infinite dimensional quotient. Then there is a sequence of Lipschitz convex functions $\left\{f_{n}\right\}$ that $\mathcal{K}$-converges $(\mathcal{W}$-converges $)$ to 0 such that $\lim _{n \rightarrow \infty} f_{n}(d)=\infty$ for all $d$ in some dense set.

(b) In any infinite dimensional (Schur) Banach space, there is a net of Lipschitz convex functions $\left\{f_{\nu}\right\}$ such that $\limsup f_{\nu}(x)=0$ for all $x$ and $\left\{f_{\nu}\right\} \mathcal{K}$-converges $(\mathcal{W}$-converges $)$ to 0 , but not uniformly on (weakly) compact sets.

Proof. In both parts of the proof we will use the following fact.

FACT A. Suppose the lsc convex functions $\left\{f_{\nu}\right\}$ converge pointwise to $f$ on a dense set of a Banach space $X$, and $f_{\nu} \geq f$ for all $\nu$. Then $\left\{f_{\nu}\right\} \mathcal{K}$-converges to $f$.

To prove Fact A, we will show for any norm compact set $\widetilde{W} \subset X \times \mathbb{R}$ and $\epsilon>0$, there is an $\bar{\nu}$ such that

$$
\widetilde{W} \cap \operatorname{epi} f_{\nu} \subset \text { epif }+\epsilon B_{X \times \mathbb{R}} \text { and } \widetilde{W} \cap \text { epif } \subset \text { epi } f_{\nu}+\epsilon B_{X \times \mathbb{R}} \text { for all } \nu \geq \bar{\nu} \text {. }
$$

Because $f_{\nu} \geq f$ for all $\nu$, the first condition is always satisfied. For the other condition, fix a compact set $W \subset X$ and $M>0$ such that $\widetilde{W} \subset W \times[-M, M]$. Now we can find $w_{1}, \ldots, w_{n} \in X$ that form an $\epsilon$-net for $W$ and a $\bar{\nu}$ with $f_{\nu}\left(w_{k}\right)<$ $f\left(w_{k}\right)+\epsilon$ for $1 \leq k \leq n$ and $\nu \geq \bar{\nu}$ (using the density hypothesis). It follows that epif $\cap \widetilde{W} \subset \operatorname{epi} f_{\nu}+\epsilon B_{X \times \mathbb{R}}$ for $\nu \geq \bar{\nu}$. This proves Fact A.

By the Eberlein-Šmulyan theorem, weakly compact sets in Schur spaces are norm compact. Hence it suffices to show the compact version only in (a) and (b).

(a) Let $X / Z$ be separable and let $\left\{\hat{x}_{n}, x_{n}^{*}\right\}_{n=1}^{\infty}$ be an M-basis of $X / Z$ with $x_{n}^{*} \in$ $Z^{\perp}$ (see [18, Proposition 1.f.3]). Fix $x_{n} \in \hat{x}_{n}$; by normalizing we may assume $\left\|x_{n}\right\|=1$ for all $n$. Define $f_{n}$ by $f_{n}(x)=2^{n}\left|x_{n}^{*}(x)\right|$. Then $f_{n}$ is Lipschitz and convex. For any fixed $x \in X$, we find $\bar{x}=z+\sum_{k=1}^{n_{0}} a_{k} x_{k}$ for some $z \in Z$ and $a_{k} \in \mathbb{R}$ such that $\|x-\bar{x}\|<\frac{\epsilon}{2}$. Now choose $N>n_{0}$ such that $\sum_{k=N}^{\infty} \frac{1}{k^{2}}<\frac{\epsilon}{2}$. Then for $\tilde{x}=\bar{x}+\sum_{k=N}^{\infty} \frac{1}{k^{2}} x_{k}$ and $n \geq N$, we have $f_{n}(\tilde{x})=\frac{2^{n}}{n^{2}} \rightarrow \infty$. On the other hand, $f_{n}(\bar{x})=0$ for all $n \geq n_{0}$ and so the sequence converges pointwise to 0 on a dense set. Using Fact $\mathrm{A}$ we conclude it $\mathcal{K}$-converges to 0 .

(b) Let $K$ be a fixed compact set that is not contained in any finite dimensional subspace of $X$. Let $\mathcal{F}$ denote all the finite subsets of $X$ directed by inclusion. For $\nu \in \mathcal{F}$, we let $\mathcal{F}_{\nu}:=\operatorname{span}\{x: x \in \nu\}$. Then $F_{\nu}$ is a closed finite dimensional subspace of $X$ and $K \backslash F_{\nu} \neq \emptyset$. So we fix $x_{\nu} \in K \backslash F$ and choose $\Lambda_{\nu} \in X^{*}$ such that $\Lambda_{\nu}(F)=0$ and $\Lambda_{\nu}\left(x_{\nu}\right)=1$. We now set $f_{\nu}:=\left|\Lambda_{\nu}\right|$. By the definition of the 
net, it is clear that $\lim _{\nu} f_{\nu}(x)=0$ for all $x \in X$. The $\mathcal{K}$-convergence follows from Fact A, while it is clear that the convergence is not uniform on $K$.

Things are actually not all that hopeless in the weakly compact case. To show this, we recall that a space is said to be sequentially reflexive if norm and Mackey convergence coincide sequentially in the dual space. In [19] it was shown that a Banach space $X$ is sequentially reflexive if and only if $X \not \supset \ell_{1}$ (by $X \not \supset \ell_{1}$ we mean $X$ does not contain an isomorphic copy of $\ell_{1}$ ). Because the manuscript [19] is unpublished, we refer the reader to Theorem 5 in the appendix of [10] for a proof of this result. We will not pursue questions of stability of $\mathcal{W}$-convergence under addition however, the next theorem has ramifications in this area (see the proof of Theorem 3.1 and the discussion following it).

Theorem 2.5. Suppose $\left\{f_{n}\right\}$ is a sequence of lsc convex functions, $f$ is a continuous convex function bounded on weakly compact sets of a Banach space not containing an isomorphic copy of $\ell_{1}$. Then $\left\{f_{n}\right\} \mathcal{W}$-converges to $f$ if and only if $\left\{f_{n}\right\}$ converges to $f$ uniformly on weakly compact sets.

Proof. The if implication follows from Lemma 1.4. For the other implication, by sequential reflexivity, the sequence $\left\{\Lambda_{k}\right\}$ in (2.1) of the proof of Theorem 2.1 does not converge weakly to 0 . Thus in this case, the proof of (b) in Theorem 2.1 can be completed without the assumption $\limsup _{n \rightarrow \infty} f_{n}(x)<\infty$ for all $x$.

As a side remark, let us mention that one can also use sequential reflexivity along with the techniques of Theorem 2.1 to obtain the following uniform boundedness variants of [12, Theorem 2.4, Theorem 2.7]. We will omit the proof.

Proposition 2.6. Suppose $X$ is a Banach space and $\left\{f_{n}\right\}$ is a family of lsc convex functions.

(a) If $X \not \supset \ell_{1}$, then $\left\{f_{n}\right\}$ is eventually uniformly bounded on each bounded set if it is eventually uniformly bounded on each weakly compact set.

(b) If weak ${ }^{*}$ and Mackey convergence coincide sequentially in $X^{*}$, then $\left\{f_{n}\right\}$ is eventually uniformly bounded above on weakly compact sets provided lim $\sup f_{n}(x)<$ $\infty$ for each $x \in X$.

\section{Applichtions of SEQUential CONVERGEnCE in DUAL topologies}

We will build on the results and techniques of Theorem 2.1 to show that uniform convergence of lsc convex functions to an affine function on certain sets can be deduced from uniform convergence on smaller sets, provided the analogous results hold for continuous linear functionals. The last portion of this section will be devoted to limiting examples.

Theorem 3.1. For a Banach space $X$, the following are equivalent.

(a) $X \not \supset \ell_{1}$.

(b) Every sequence of lsc convex functions that converges uniformly on weakly compact sets to a continuous affine function $\phi$ must converge uniformly on bounded sets to $\phi$.

(c) Every sequence of lsc convex functions that $\mathcal{W}$-converges to an affine function $\phi$ must converge uniformly on bounded sets to $\phi$. 
Proof. (a) $\Rightarrow$ (b): If $\left\{g_{n}\right\}$ is a sequence of lsc convex functions converging to $\phi$ uniformly on weakly compact sets, then letting $f_{n}=g_{n}-\phi,\left\{f_{n}\right\}$ converges uniformly to 0 on weakly compact sets. Suppose $\left\{f_{n}\right\}$ does not converge to 0 uniformly on bounded sets, then by convexity the convergence fails from above since $f_{n}(0) \rightarrow 0$. Thus for some ball $K B_{X}$ and $\left\{x_{n}\right\} \subset K B_{X}$, for a subsequence, we have

$$
f_{k}\left(x_{k}\right)>\epsilon \text { for all } k \text {. }
$$

Now consider $C_{k}=\left\{x: f_{k}(x) \leq \epsilon\right\}$. As we did in Theorem 2.1, we obtain $\Lambda_{k} \in S_{X^{*}}$ so that (2.1) holds. Using sequential reflexivity we find a weakly compact set $W$ and a subsequence $\left\{\Lambda_{j}\right\}$ such that $\sup _{W} \Lambda_{j}>K$ for all $j$. Choosing $w_{j} \in W$ such that $\Lambda_{j}\left(w_{j}\right)>K \geq\left\|x_{j}\right\| \geq \Lambda_{j}\left(x_{j}\right)$ it follows from (2.1) that $w_{j} \notin C_{j}$. Hence $f_{j}\left(w_{j}\right)>\epsilon$ and so the convergence is not uniform on $W$. This proves (a) $\Rightarrow(\mathrm{b})$.

$(\mathrm{b}) \Rightarrow(\mathrm{c})$ : Because the condition applies to continuous linear functionals, $X$ is sequentially reflexive and so $X \not \supset \ell_{1}$. Therefore, we can use Theorem 2.5 to obtain (c).

(c) $\Rightarrow(\mathrm{a})$ : Use Lemma 1.3 and sequential reflexivity.

We didn't explicitly need results on stability of $\mathcal{W}$-convergence under addition in the above theorem because Theorem 2.5 implicitly contains special cases of such results (because uniform convergence on weakly compact sets is stable under addition). For some stability results on Attouch-Wets convergence and their applications see [9]; see also [17, Section 4]. A natural situation where the above theorem can be used is in questions of differentiability. First, recall that a function $f$ is said to be Fréchet (resp. weak Hadamard, Gateaux) differentiable at $x$, if there is an $f^{\prime}(x) \in X^{*}$ such that

$$
\lim _{t \rightarrow 0} \frac{f(x+t h)-f(x)}{t}=f^{\prime}(x)(h)
$$

uniformly on bounded sets (resp. weakly compact sets, singletons).

Corollary 3.2. Let $X$ be a Banach space. Then:

(a) $\left(\left[6\right.\right.$, Theorem 2]) $X \not \supset \ell_{1}$ if and only if Fréchet and weak Hadamard differentiability coincide for all lsc convex functions on $X$.

(b) $X \not \supset \ell_{1}$ if and only if norm-Mackey and norm-norm continuity, equivalently lower semicontinuity, coincide for every maximal monotone operator $T: X \rightarrow X^{*}$. This remains valid for minimal weak ${ }^{*}$-cuscos $T: \Omega \rightarrow X^{*}$ whenever $: \Omega$ is metric.

(c) Suppose $X \not \supset \ell_{1}$ and $\left\{f_{n}\right\}$ is a sequence of weakly continuous convex functions that decreases pointwise to an affine function $\phi$. Then $\left\{f_{n}\right\}$ converges uniformly to $\phi$ on bounded sets.

Proof. (a) Suppose $X \not \supset \ell_{1}$. The difference quotients $n\left[f\left(x+\frac{1}{n}(\cdot)\right)-f(x)\right]$ converge uniformly on weakly compact sets to the continuous linear functional $f^{\prime}(x)(\cdot)$ provided $f$ is weak Hadamard differentiable at $x$. Thus using Theorem 2.1(b) the convergence is uniform on bounded sets which shows Fréchet differentiability. This was derived in [10, Theorem 2] using sequential reflexivity and Šmulyan's criterion. For the converse implication, see [10, Theorem 2].

(b) (Sketch) We include this as a further application of sequential reflexivity that was not included in [10]. We refer the reader to [20] for concepts not introduced 
here. First, any maximal monotone is a minimal weak* cusco. Second, if a minimal weak*-cusco is lsc at a point $t_{0}$, then it is single valued and continuous there (see e.g. [20]). Now let $t_{n} \rightarrow t_{0}$ and let $x_{n}^{*} \in T\left(t_{n}\right)$. By Mackey continuity, $\left\{x_{n}^{*}\right\}$ converges Mackey to $x_{0}^{*}$. Applying sequential reflexivity yields the conclusion. The converse follows from [10, Theorem 2].

(c) This is a direct consequence of Dini's theorem and Theorem 3.1.

Even though continuous convex functions are weakly lsc, part (c) of the above theorem can fail if one doesn't assume weak continuity. For example, consider $X=c_{0}$ and $f_{n}(x):=\sup \{|x(k)|: k>n\}$ where $x:=\{x(k)\}_{k=1}^{\infty}$. Then $\left\{f_{n}\right\}$ decreases pointwise to 0 , but $\left\{f_{n}\right\}$ does not converge uniformly to 0 on the weakly compact set $\left\{e_{n}\right\}_{n=1}^{\infty} \cup\{0\}$. In Corollary 3.4, we will show there are spaces where such examples cannot exist. First we describe sets in Banach spaces on which lsc convex functions converge uniformly to 0 provided they converge pointwise to 0 .

Theorem 3.3. For a set $S$ in a Banach space $X$, the following are equivalent.

(a) Every sequence of lsc convex functions eventually bounded above at each point that $\mathcal{P}$-converges to a continuous affine function $\phi$, must converge uniformly to $\phi$ on $S$.

(b) Every sequence of lsc convex functions converging pointwise on $X$ to a continuous affine function $\phi$ must converge uniformly to $\phi$ on $S$.

(c) Every sequence in $X^{*}$ converging weak* to 0 must converge uniformly to 0 on $S$.

(d) Every continuous convex function is bounded on $S$.

Proof. Notice that all conditions imply $S$ is a bounded set. The equivalence of (c) and $(d)$ is stated for comparison and it follows from [12, Lemma 2.1 and Lemma 2.6]. Lemma 1.4 shows (a) $\Rightarrow$ (b) while (b) $\Rightarrow$ (c) is trivial, so we show (c) $\Rightarrow$ (a).

We will suppose (a) holds and proceed by contradiction. Let $\left\{g_{n}\right\} \mathcal{P}$-converge to $\phi$ and suppose $\left\{g_{n}(x)\right\}$ is eventually bounded above for each $x$. According to Theorem 2.1, we know $\left\{g_{n}\right\}$ converges pointwise to $\phi$ but we may suppose the convergence is not uniform on $S$. If $f_{n}=g_{n}-\phi$, then $\left\{f_{n}\right\}$ converges pointwise to 0 , but not uniformly on $S$. As in Theorem 3.1, after passing to a subsequence, we choose an $\epsilon>0$ such that $f_{k}\left(x_{k}\right)>\epsilon$ for some sequence $\left\{x_{k}\right\} \in S$. For $C_{k}=\left\{x: f_{k}(x) \leq \epsilon\right\}$, we have $\Lambda_{k} \in S_{X^{*}}$ such that (2.1) holds. The pointwise convergence to 0 along with a standard Baire category with convexity argument (as in Step 2 of Theorem 2.1) show there are $N \in \mathbb{N}$ and $\delta>0$ such that $f_{k}(x) \leq \frac{\epsilon}{2}$ for all $k \geq N$ and $\|x\| \leq \delta$. Hence $\Lambda_{k}\left(x_{k}\right) \geq \delta$ for all $k$. The hypothesis now implies $\left\{\Lambda_{k}\right\}$ does not converge weak* to 0 . Thus we find $h$ such that $\Lambda_{k}(h)>\sup \{\|x\|: x \in S\}$ for infinitely many $k$. Finally one uses $(2.1)$ to show $\left\{f_{k}(h)\right\}$ does not converge to 0 to obtain a contradiction.

Recall that Proposition 2.4(a) shows the eventual pointwise boundedness assumption is needed in (a). Also, it is a fairly easy exercise to show that any set $S$ satisfying (b) is relatively norm compact provided $B_{X^{*}}$ is $w^{*}$-sequentially compact. However, in certain large spaces, $S$ need not be relatively norm compact as is seen from the next corollary and the comments following it.

Corollary 3.4. Let $X$ be a Banach space. Then the following are equivalent.

(a) Weak* and Mackey convergence coincide sequentially in $X^{*}$. 
(b) A sequence of lsc convex functions $\left\{f_{n}\right\}$ converges uniformly to a continuous affine function $\phi$ on weakly compact sets provided $\left\{f_{n}\right\}$ converges pointwise to 0 on $X$.

(c) Weak Hadamard and Gateaux differentiability coincide for all lsc convex functions on $X$.

Proof. Theorem 3.3 immediately implies (a) $\Rightarrow(\mathrm{b})$ and $(\mathrm{b}) \Rightarrow(\mathrm{c})$ follows as in Corollary 3.2(a). One needs to invoke [10, Proposition 1] to prove (c) $\Rightarrow$ (a).

In addition to the trivial examples of Schur spaces, any Grothendieck space with the Dunford-Pettis property, in particular $\ell_{\infty}$, satisfies the hypothesis of Corollary 3.4 (see [15]). For further discussion on such spaces, see [11].

We should emphasize that the results of this section are only valid for sequences of functions. The corresponding questions for nets are not interesting because they would hold for continuous linear functionals and then the underlying bornologies would be the same. However, it is natural to ask whether one can replace the limit 0 with an arbitrary continuous convex function or whether the results would be valid for differences of convex functions. The next proposition gives a strong negative answer to these questions with respect to Corollary 3.4, while in the case of Theorem 3.1 we are only able to answer the second question.

Proposition 3.5. (a) If a Banach space is not reflexive, then there are sequences of 1-Lipschitz convex functions $\left\{f_{n}\right\},\left\{g_{n}\right\}$ such that $\left\{f_{n}-g_{n}\right\}$ converges to 0 uniformly on weakly compact sets but not on bounded sets

(b) If $X$ fails the Schur property, then there are norms $\|\cdot\|_{n}$ such that $\frac{1}{2}\|\cdot\| \leq$ $\|\cdot\|_{n} \leq\|\cdot\|$ and $\|\cdot\|_{n} \rightarrow\|\cdot\|$ pointwise but not uniformly on weakly compact sets.

Proof. (a) In any nonreflexive space there are a sequence $\left\{x_{n}\right\} \subset S_{X}$ and a $\delta>0$ such that $\left\|x_{n}-x_{m}\right\|>2 \delta$ for all $n \neq m$ and $\left\{y_{n}\right\}$ has no weakly convergent subsequence provided $\left\|y_{n}-x_{n}\right\|<\delta$ for each $n$ (see [11, Lemma 2.2]). Let $g_{n}(x):=$ $\left\|x-x_{n}\right\|-\delta$ and $f_{n}(x):=\max \left\{g_{n}(x), 0\right\}$. For $h_{n}:=f_{n}-g_{n}$ we have $h_{n}(x)=\delta-$ $\left\|x-x_{n}\right\|$ if $x \in B_{\delta}\left(x_{n}\right):=\left\{x:\left\|x-x_{n}\right\|<\delta\right\}$ and $h_{n}(x)=0$ otherwise. Now $h_{n}\left(x_{n}\right)=\delta$ and so $\left\{h_{n}\right\}$ does not converge to 0 uniformly on $B_{X}$. Suppose $W$ is weakly compact. Then $W$ can intersect only finitely many $B_{\delta}\left(x_{n}\right)$. Indeed, if $w_{k} \in W \cap B_{\delta}\left(x_{n_{k}}\right)$, then $\left\{w_{k}\right\}$ could not have a weakly convergent subsequence; this cannot happen according to the Eberlein-Šmulyan theorem and the weak compactness of $W$. Therefore there is an $N$ such that $W \cap B_{\delta}\left(x_{n}\right)=\emptyset$ for all $n \geq N$ and so $h_{n}(w)=0$ for all $n \geq N$ and all $w \in W$.

(b) Consider $w_{n} \in X$ such that $\left\{w_{n}\right\} \stackrel{w}{\rightarrow} 0$ but $\left\|w_{n}\right\|=1$ for all $n$. Define $\|\cdot\|_{n}$ by $\|x\|_{n}=\frac{1}{2}\|x\|+\frac{1}{2} d\left(x, W_{n}\right)$ where $W_{n}$ is the one dimensional subspace spanned by $w_{n}$. Now $d\left(x, W_{n}\right) \leq\|x\|$ for all $n$ and so it is clear $\frac{1}{2}\|\cdot\| \leq\|\cdot\|_{n} \leq\|\cdot\|$ for all $n$. Moreover, $\left\|w_{n}\right\|_{n}=1 / 2$ for all $n$ and so the convergence is not uniform on weakly compact sets. On the other hand, for a fixed $x \in X$, we fix $v_{n} \in W_{n}$ such that $\left\|x-v_{n}\right\|=d\left(x, W_{n}\right)$. By the triangle inequality, $\left\|v_{n}\right\| \leq 2\|x\|$ and so $\left\{v_{n}\right\} \stackrel{w}{\rightarrow} 0$. By the weak lower semicontinuity of the norm, $\liminf _{n \rightarrow \infty}\left\|x-v_{n}\right\| \geq\|x\|$. Thus $\liminf _{n \rightarrow \infty} d\left(x, W_{n}\right) \geq\|x\|$ and we are done.

The converse of Proposition 3.5(a) is clearly true. In fact, the existence of functions as in (a) easily allows one to construct the sequence given by [11, Lemma $2.2]$. 
Let us also mention that the original motivation for Borwein and Beer [6] to consider when Mackey and norm convergence coincide sequentially in the dual space was to show in such spaces that continuous linear functionals whose epigraphs (or equivalently graphs) converge Mosco must also converge in norm, i.e. uniformly on bounded sets. In contrast to this, the following remark shows that " $\mathcal{W}$ convergence" cannot be replaced by Mosco convergence in Theorem 3.1. Let us first recall $\left\{f_{n}\right\}$ converges Mosco to $f$ if for each $x \in X$ there is a sequence $\left\{x_{n}\right\} \rightarrow x$ with $\left\{f_{n}\left(x_{n}\right)\right\} \rightarrow f(x)$ and if $\liminf _{n \rightarrow \infty} f_{n}\left(x_{n}\right) \geq f(x)$ whenever $\left\{x_{n}\right\}$ converges weakly to $x$.

Remark 3.6. (a) Let $X$ be any Banach space not containing $\ell_{1}$. Then there is a sequence of 1-Lipschitz convex functions that converges Mosco and pointwise to 0 but not does not $\mathcal{W}$-converge to 0 .

(b) $\mathcal{W}$-convergence and Mosco convergence coincide for every sequence of lsc convex functions on a Banach space if and only if the space is Schur.

Proof. (a) By the Josefson-Nissenzweig theorem (see [14]) there is a sequence $\left\{\Lambda_{n}\right\}$ $\subset S_{X^{*}}$ that converges weak* to 0 . According to sequential reflexivity, this sequence does not converge Mackey to 0 . Defining $f_{n}(x):=\left|\Lambda_{n}(x)\right|$ one can easily check that the statement of the remark is satisfied.

(b) In a Schur space, it follows directly that $\mathcal{W}$-convergence, Painlevé-Kuratowski convergence and Mosco convergence all coincide. If $X$ is not Schur, consider the functions constructed in Proposition 3.5(b), and check that the convergence is Mosco. Indeed, the first condition is trivial by pointwise convergence. For the second condition, if $\left\{x_{n}\right\} \stackrel{w}{\rightarrow} x$, then with $v_{n} \in W_{n}$ such that $\left\|v_{n}-x_{n}\right\|=d\left(x_{n}, W_{n}\right)$ we have $\left\{v_{n}\right\} \stackrel{w}{\rightarrow} 0$ and so $\liminf _{n \rightarrow \infty}\left\|v_{n}-x_{n}\right\| \geq\|x\|$.

Our last example demonstrates there is no hope of obtaining a general result showing $\mathcal{W}$-convergence implies Attouch-Wets convergence on spaces not containing $\ell_{1}$.

Example 3.7. On $c_{0}$ one can construct a sequence of Lipschitz convex functions $\left\{f_{n}\right\}$ that $\mathcal{W}$-converges to a lsc convex function $f$ but does not converge AttouchWets to $f$.

Proof. For $\Lambda \in \ell_{1}$, let $\Lambda(k)$ denote the $k$-th coordinate of $\Lambda$. Now define $f_{n}$ by

$$
f_{n}(x):=\sup \left\{\Lambda(x): \Lambda \in \ell_{1}, \Lambda(k) \geq 0,\|\Lambda\|_{1} \leq n, \sum_{k=1}^{n} \Lambda(k) \geq 1\right\} .
$$

Let $c_{0}^{-}:=\left\{x \in c_{0}: x(k) \leq 0\right.$ for all $\left.k\right\}$ where $x(k)$ denotes the $k$-th coordinate of $x$. We let $f$ denote the indicator function of $c_{0}^{-}$. Now $f_{n} \leq f$ for all $n$, therefore to establish $\mathcal{W}$-convergence (using the Eberlein-S̈mulyan theorem) we need only show for each $\epsilon>0$, and subsequences $\left\{f_{k}\right\},\left\{w_{k}\right\}$ with $\left\{f_{k}\left(w_{k}\right)\right\}$ bounded and $\left\{w_{k}\right\}$ converging weakly to some $w$, there is an $N$ with $f_{k}\left(w_{k}\right) \in$ epi $f+\epsilon B_{c_{0} \times \mathbb{R}}$ for all $k \geq N$. Because $\left\{f_{k}\left(w_{k}\right)\right\}$ is bounded, we know there is an $n_{1}$ with $w_{k} \in c_{0}^{-}+\epsilon B_{c_{0}}$ for all $k \geq n_{1}$. Because $\left\{w_{k}\right\}$ converges weakly, we know there is an $N \geq n_{1}$ such that $w_{k}(N)>-\epsilon$ for all $k \geq N$. Now fix $\Lambda_{k} \in \ell_{1}$ such that $\Lambda_{k}(N)=1$ and $\Lambda_{k}(j)=0$ otherwise. Then $\Lambda_{k}\left(w_{k}\right)>-\epsilon$. Therefore, $f_{k}\left(w_{k}\right)>-\epsilon$ for $k \geq N$ and so $\left(w_{k}, f_{k}\left(w_{k}\right)\right) \in$ epi $f+\epsilon B_{c_{0} \times \mathbb{R}}$ for all $k \geq N$. This shows $\left\{f_{n}\right\} W$-converges to $f$. 
To see that $\left\{f_{n}\right\}$ does not converge Attouch-Wets to $f$, consider $\left\{x_{n}\right\}$ defined by $x_{n}(k)=-1$ if $k \leq n$ and $x_{n}(k)=0$ otherwise. Then $f_{n}\left(x_{n}\right)=-1$ while $f \geq 0$ and so the convergence cannot be Attouch-Wets.

Unfortunately we have not answered the following questions. If $X \not \supset \ell_{1}$, does uniform convergence on weakly compact sets of a sequence of lsc convex functions to a continuous convex function imply the convergence is uniform on bounded sets? What if the convex limit function is Lipschitz or weakly continuous? What if $f_{n}(x):=n\left[f\left(x_{0}+\frac{x}{n}\right)-f\left(x_{0}\right)\right]$ where $f$ is convex continuous but not differentiable (compare Corollary 3.2)? Observe it would be interesting if a positive result holds for (certain) weakly continuous limit functions because such a result in conjunction with Dini's theorem would show sequence of lsc convex functions that increases pointwise to such a (certain) weakly continuous convex function would converge uniformly on bounded sets. Note that Dini's theorem fails if the limit is not weakly continuous. For example, $f_{n}(x):=\max \{|x(k)|: k \leq n\}$ are weakly continuous and increase pointwise to the usual norm on $c_{0}$ but the sequence does not converge uniformly on weakly compact sets.

Note added in proof. The authors have recently constructed examples providing negative answers to the above questions for Lipschitz convex limit functions. See Convex functions on 'sequentially reflexive' Banach spaces, CECM Preprint \#95:050, Simon Fraser University, 1995.

\section{REFERENCES}

1. H. Attouch and R. Wets, Quantitative stability of variational systems: I. The epigraphical distance, Trans. Amer. Math. Soc. 328 (1991), 695-730. MR 92c:90111

2. G. Beer, Convergence of linear functionals and their level sets, Arch. Math. 55 (1989), 285292. MR 90i: 46018

3. Topologies on Closed and Closed Convex Sets, Kluwer Academic Publishers, The Netherlands, 1993. MR 94:10

4.. _ Wijsman convergence of convex sets under renorming, Nonlinear Anal. 22 (1994), 207-216. MR 94m:46021

5. _ Lipschitz regularizations and the convergence of convex functions, Numer. Funct. Anal. Optim. 15 (1994), 31-46. MR 94m:49018

6. G. Beer and J. Borwein, Mosco and slice convergence of level sets and graphs of linear functionals, J. Math. Anal. Appl. 175 (1993), 53-67. MR 94i:46031

7. G. Beer and A. DiConcilio, Uniform continuity on bounded sets and the Attouch-Wets topology, Proc. Amer. Math. Soc. 112 (1991), 235-243. MR 91h:54013

8. G. Beer and R. Lucchetti, Convex optimization and the epi-distance topology, Trans. Amer. Math. Soc. 327 (1991), 795-813. MR 92a:49018

9. 느 The epi-distance topology: continuity and stability results with applications to convex optimization problems, Math. Oper. Res. 17 (1992), 715-726. MR 93k:49011

10. J. Borwein and M. Fabian, On convex functions having points of Gateaux differentiability which are not points of Fréchet differentiability, Canad. J. Math. 45 (1993), 1121-1134. MR 94i:46018

11. J. Borwein, M. Fabian and J. Vanderwerff, Locally Lipschitz functions and bornological derivatives, CECM Information document 93-012, Simon Fraser University.

12. J. Borwein, S. Fitzpatrick and J. Vanderwerff, Examples of convex functions and classifications of normed spaces, J. Convex Analysis 1 (1994), 61-73. CMP 95:11

13. J. Borwein and J. Vanderwerff, Convergence of Lipschitz regularizations of convex functions, J. Funct. Anal. 128 (1995), 139-162. CMP 95:08

14. J. Diestel, Sequences and Series in Banach Spaces, Graduate Texts in Mathematics, vol. 92 Springer-Verlag, Berlin, New York, and Tokyo, 1984. MR 85i:46020 
15. J. Diestel and J.J. Uhl, Jr, Vector Measures, AMS Mathematical Surveys 15 (1977), American Mathematical Society, Providence, RI. MR 56:12216

16. J. Lahrache, Stabilité et convergence dans les espaces non reflexives, Sém. Anal. Convexe 21 (1991), exposé no. 10, 10.1-10.50. MR 92m:49021

17. A.B. Levy, R. Poliquin and L. Thibault, Partial extensions of Attouch's theorem with applications to proto-derivatives of subgradient mappings, Trans. Amer. Math. Soc. 347 (1995), 1269-1294. MR 95k:49035

18. J. Lindenstrauss and L. Tzafriri, Classical Banach Spaces I, Springer-Verlag, Berlin, Heidelberg, and New York, 1977. MR 58:17766

19. P. Ørno, Banach Bulletin Board, 1991.

20. R.R. Phelps, Convex Functions, Monotone Operators and Differentiability, Lecture Notes in Mathematics, vol. 1364, Springer-Verlag, 1989. MR 90g:46063

21. G. Salinetti and R. Wets, On the relationship between two types of convergence for convex functions, J. Math. Anal. Appl. 60 (1977), 211-226. MR 57:18828

22. Y. Sonntag and C. Zalinescu, Set convergences. An attempt of classification, Trans. Amer. Math. Soc. 340 (1993), 199-226. MR 94a:54006

Department of Mathematics and Statistics, Simon Fraser University, Burnaby, BC, CANADA V5A 1S6

E-mail address: jborwein@cecm.sfu.ca

Department of Mathematics, Walla Walla College, College Place, Washington 99324

E-mail address: vandjo@wwc.edu 